\title{
Índice de massa corporal: tendência secular em crianças e adolescentes brasileiros
}

\author{
Body mass index: secular trends in children and adolescents \\ from Brazil
}

\author{
Gabriel Gustavo Bergmann ${ }^{1,2}$ \\ Mauren Lúcia de Araújo Bergmann ${ }^{2,3}$ \\ Eraldo dos Santos Pinheiro 2 \\ Rodrigo Baptista Moreira ${ }^{1,2}$ \\ Alexandre Carriconde Marques 2 \\ Daniel Carlos Garlipp 2 \\ Adroaldo Gaya 2
}

1 Universidade Luterana do Brasil. Curso de Educação Física. São Jerônimo, RS. Brasil.

2 Universidade Federal do Rio Grande do Sul. Projeto Esporte Brasil. Centro de Excelência Esportiva. Programa de Pós-Graduação em Ciências do Movimento Humano. Porto Alegre, RS. Brasil.

3 Universidade Luterana do Brasil. Programa de Pós-Graduação em Saúde Coletiva. Porto Alegre, RS. Brasil.

Recebido em 18/12/07 Aprovado em 24/07/08
Resumo - O objetivo deste estudo foi comparar a distribuição do índice de massa corporal (IMC) de crianças e adolescentes brasileiros avaliados em 1989, com crianças e adolescentes avaliados em 2004/2005. Foi comparada a distribuição do IMC de crianças e adolescentes de 07 a 17 anos, avaliados em dois grandes levantamentos realizados em todo o Brasil em 1989 e em 2004/2005. As amostras foram compostas por 16.012 (7.908 meninas) e por 54.574 (24.144 meninas), no primeiro e segundo levantamento, respectivamente. A distribuição dos resultados foi feita a partir dos percentis 15 (P15), 50 (P50) e 85 (P85). Os resultados demonstraram que no P15, praticamente, não houve mudanças nos valores de IMC, no P50 e P80, por outro lado, ocorreram aumentos, sendo nos meninos ao longo de todas as idades, e nas meninas até em torno dos 11/12 anos. Seguindo os mesmos resultados de outros estudos, também no Brasil parece estar havendo aumento nos valores de IMC de crianças e adolescentes com o passar dos anos, aumentando a prevalência de sobrepeso e obesidade nesta faixa etária.

Palavras-chave: Índice de massa corporal; Crianças; Adolescentes; Brasil.

Abstract - The aim of this study was to compare the distribution of body mass index (BMI) between Brazilian children and adolescents aged 7 to 17 years evaluated in 1989 and in 2004/2005. BMI distribution was compared using two large surveys conducted throughout Brazil in 1989 and 2004/2005. The samples consisted of 16,012 (7908 girls) and 54,574 (24,144 girls) children and adolescents in the first and second survey, respectively. BMI distribution was evaluated using the 15th (P15), 50th (P50), and 85th percentiles (P85). The results showed practically no changes in $P 15$, whereas P50 and P85 increased in boys along all ages and in girls until 11/12 years of age. The increase in P85 was higher than that in P50 in both genders. As observed in other countries, in Brazil BMI seems to be increasing in children and adolescents with age, with a consequent increase in the prevalence of overweight and obesity in this age group.

Key words: Body mass index; Children; Adolescents; Brazil. 


\section{INTRODUÇÃO}

A obesidade tem atingido proporções preocupantes em todo o mundo, afetando cerca de $55 \%$ dos adultos nos EUA ${ }^{1}$. Esta epidemia não se restringe apenas aos adultos, havendo também um exacerbado aumento na prevalência da obesidade em crianças em idade pré-escolar ${ }^{2}$ e em crianças e adolescentes de 6 a 17 anos $^{3}$. Este aumento nos níveis de gordura de crianças e adolescentes tem acarretado uma série de problemas de saúde, que até pouco tempo era associado apenas aos adultos, tais como hipertensão arterial, elevados níveis de lipídeos e glicose sanguínea, e intolerância à glicose $e^{4-6}$.

No Brasil, o estudo do sobrepeso e obesidade em crianças e adolescentes tem sido investigado em diferentes regiões. Abrantes et al. ${ }^{7}$, estudando crianças da região Sudeste e Nordeste do Brasil, relataram uma prevalência de obesidade $12 \%$ e $8 \%$, respectivamente. Em estudo realizado com crianças de Brasília, foi encontrada prevalência de cerca de $20 \%$ de sobrepeso e obesidade ${ }^{8}$. Com resultados próximos aos encontrados no estudo anterior, Bergmann et al. ${ }^{9}$, analisando crianças e adolescentes do estado do Rio grande do Sul, encontraram uma prevalência de sobrepeso e obesidade de cerca de 20\%, e Terres et al. ${ }^{10}$, estudando adolescentes de Pelotas/RS encontraram prevalências de sobrepeso e obesidade $20,9 \%$ e $5 \%$, respectivamente.

Preocupados com esta situação, pesquisadores de vários países têm realizado estudos comparativos acerca do estado nutricional de crianças e adolescentes ao longo do tempo. A maioria destes estudos encontrou aumentos tanto nos níveis médios de IMC, quanto na proporção de indivíduos classificados como acima do peso e obesos ${ }^{11-13}$. No Brasil, ainda não foi realizado um estudo desta natureza com uma amostra que representasse crianças e adolescentes de todo o país. Com o intuito de disponibilizar esta informação, o objetivo deste estudo é comparar a distribuição do índice de massa corporal (IMC) de crianças e adolescentes avaliados em 1989, com crianças e adolescentes avaliados em 2004/2005.

\section{PROCEDIMENTOS METODOLÓGICOS}

Este estudo faz parte de uma série de trabalhos que estão sendo desenvolvido pelo Projeto Esporte Brasil (PROESP-BR - ver www.proesp.ufrgs.br). Realizado a partir de dois grandes levantamentos transversais, caracteriza-se como descritivo, apresentando a distribuição dos valores de IMC da população brasileira de 7 a 17 anos, nos anos de 1989 e de 2004/2005. A primeira foi feita com base nos resultados da Pesquisa Nacional sobre Saúde e Nutrição (PNSN), realizada pelo Instituto Brasileiro de Geografia e Estatística (IBGE) e pelo Instituto Nacional de Alimentação e Nutrição (INAN) em 1989 (para maiores detalhamentos acerca dos procedimentos de seleção da amostra e dos materiais e métodos utilizados para as medidas, ver INAN ${ }^{14}$ e ANJOS et al. ${ }^{15}$ ). A segunda foi feita com base nos resultados do levantamento realizado nos anos de 2004 e 2005 pelo PROESP-BR, utilizando uma amostra não aleatória por conveniência constituída através dos seguintes procedimentos: (1) Houve o convite do Ministro de Estado do Esporte aos secretários estaduais e municipais de esporte e educação no qual foi apresentado o Projeto Esporte Brasil e foram planejadas as formas de ação; (2) Foi realizado em Brasília um curso de formação para avaliadores de todos os estados e do distrito federal; (3) foi criado um site com todas as informações sobre os procedimentos de avaliação (www.proesp.ufrgs.br); (4) a partir das secretarias estaduais e municipais de esporte e educação, foram encaminhados convites para participação das escolas da rede pública e privada; (5) realizou-se no dia 5 de setembro de 2004 em todas as capitais brasileiras o dia nacional de avaliação; (6) após o dia 5 de setembro as equipes de avaliadores previamente treinadas concentraram-se em visitas as escolas (urbanas e rurais) conforme cronograma previamente estabelecido pelos coordenadores regionais. Portanto, a avaliação prosseguiu nas escolas brasileiras até outubro do ano de 2005; (7) Todos os dados foram digitados e enviados pelo site PROESP-BR para o Centro de Processamento de Dados da UFRGS, onde se constituiu o Banco de Dados PROESP-BR. A descrição dos resultados foi feita a partir dos valores dos percentis 15 (P15), 50 (P50) e 80 (P80). Para o cálculo dos percentis estratificados por sexo e idade, foi utilizado o programa estatístico SPSS for Windows versão 10.0. O PROESP-BR foi aprovado pelo Comitê de Ética em Pesquisas da Universidade Federal do Rio Grande do Sul, na reunião número 11, ata número 91 de 09/08/2007.

\section{RESULTADOS}

As distribuições dos valores de IMC de meninos e meninas brasileiras de 7 a 17 anos, nos estudos realizados pelo INAN, (1990) e pelo PROESP-BR 
Tabela 1. Distribuição dos valores de índice de massa corporal $\left(\mathrm{kg} / \mathrm{m}^{2}\right)$ da população brasileira masculina e feminina de 7 a 17 anos em 1989 e em 2004/2005.

\begin{tabular}{|c|c|c|c|c|c|c|c|c|c|c|c|c|c|c|c|c|}
\hline \multirow[b]{3}{*}{ idade } & \multicolumn{8}{|c|}{ MENINOS } & \multicolumn{8}{|c|}{ MENINAS } \\
\hline & \multicolumn{4}{|c|}{ INAN (1990) } & \multicolumn{4}{|c|}{ PROESP-BR 2004/05 } & \multicolumn{4}{|c|}{ INAN (1990) } & \multicolumn{4}{|c|}{ PROESP-BR 2004/05 } \\
\hline & $\mathrm{n}$ & P15 & P50 & P85 & $\mathrm{n}$ & P15 & P50 & P85 & $\mathrm{n}$ & P15 & P50 & P85 & $\mathrm{n}$ & P15 & P50 & P85 \\
\hline 8 & 803 & 14,3 & 15,9 & 17,2 & 538 & 14,5 & 16,2 & 19,5 & 718 & 14,2 & 15,6 & 17,4 & 453 & 14,6 & 16,6 & 19,6 \\
\hline 9 & 767 & 14,5 & 15,9 & 17,5 & 1127 & 14,1 & 16,4 & 20,1 & 802 & 14,4 & 15,8 & 17,8 & 934 & 14,0 & 16,2 & 20,0 \\
\hline 11 & 782 & 15,1 & 16,5 & 18,7 & 4931 & 14,9 & 17,1 & 20,9 & 768 & 15,1 & 16,8 & 19,8 & 4505 & 14,9 & 17,4 & 20,9 \\
\hline 12 & 721 & 15,4 & 16,9 & 19,1 & 5648 & 15,3 & 17,5 & 21,3 & 757 & 15,7 & 17,9 & 20,9 & 4666 & 15,7 & 18,2 & 21,7 \\
\hline 13 & 732 & 16,0 & 17,7 & 20,1 & 5543 & 15,9 & 18,2 & 21,7 & 713 & 16,7 & 19,0 & 22,2 & 4075 & 16,5 & 18,9 & 22,1 \\
\hline 14 & 742 & 16,4 & 18,3 & 20,8 & 4771 & 16,7 & 18,9 & 22,2 & 699 & 17,3 & 20,0 & 23,3 & 3306 & 17,2 & 19,7 & 22,9 \\
\hline
\end{tabular}

em 2004/2005, são apresentadas na tabela 1 e ilustrados nas figuras 1 e 2 . Analisando os resultados referentes aos meninos, observa-se que a medida em que os percentis vão subindo, a diferença entre o IMC do levantamento realizado pelo INAN e do levantamento realizado pelo PROESP-BR vai aumentando. No P15, as curvas praticamente se sobrepõem ao longo das idades. No P50, os valores apresentados pelos meninos do levantamento realizado pelo PROESP-BR são superiores ao longo de todas as idades em aproximadamente, $0,5 \mathrm{~kg} / \mathrm{m}^{2}$. No percentil mais alto (P85), as diferenças são de aproximadamente $2 \mathrm{~kg} / \mathrm{m}^{2}$ até em torno dos 13 anos, havendo uma redução nesta diferença até os 16 anos, quando a distância entre as duas curvas volta a aumentar, indicando uma estabilização nos valores dos meninos do levantamento realizado pelo INAN, e uma tendência a manter aumentos nos valores dos meninos do levantamento do PROESP-BR.

Diferentemente do observado nos meninos, nas meninas as diferenças nos valores de IMC apresentados entre os levantamentos de INAN e do PROESP-BR são menos marcadas, principalmente, a partir dos 12/13 anos, quando as curvas praticamente se sobrepõem independentemente do percentil analisado. Apenas dos 7 aos 12 anos observa-se algumas diferenças entre os levantamentos nos percentis 50 e 85 . No P50, há diferenças aos 7 e 8 anos, girando em torno de $1 \mathrm{~kg} / \mathrm{m}^{2}$, diminuindo aos 9 anos para cerca de $0,5 \mathrm{~kg} / \mathrm{m}^{2}$ e mantendo-se desta forma até os 11 anos. No P85, é observado a mesma diferença entre os levantamentos. Todavia, a magnitude desta diferença praticamente dobra e a aproximação entre as duas curvas acontece de forma mais gradual.

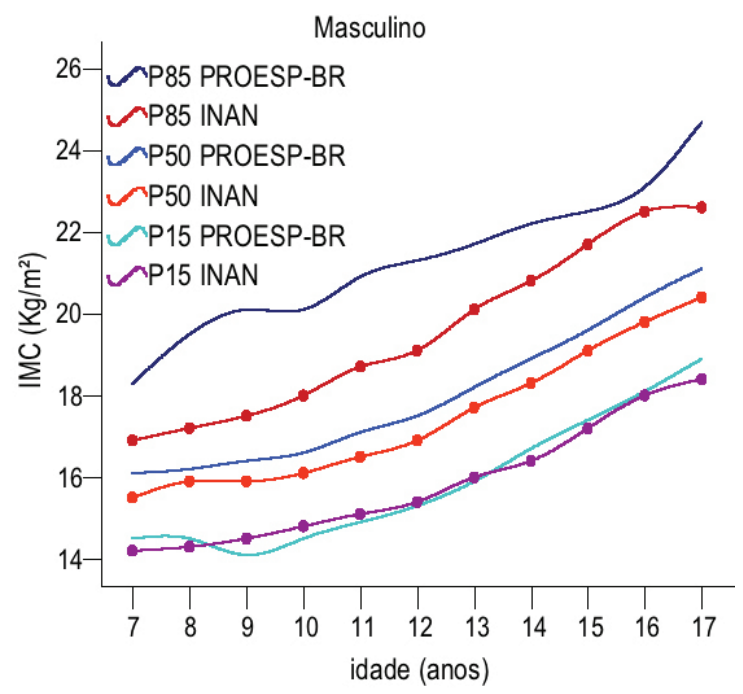

Figura 1. Distribuição dos valores (P15, P50 e P85) de índice de massa corporal $\left(\mathrm{kg} / \mathrm{m}^{2}\right)$ da população brasileira masculina de 7 a 17 anos, em 1989 (INAN) e em 2004/2005 (PROESP-BR).

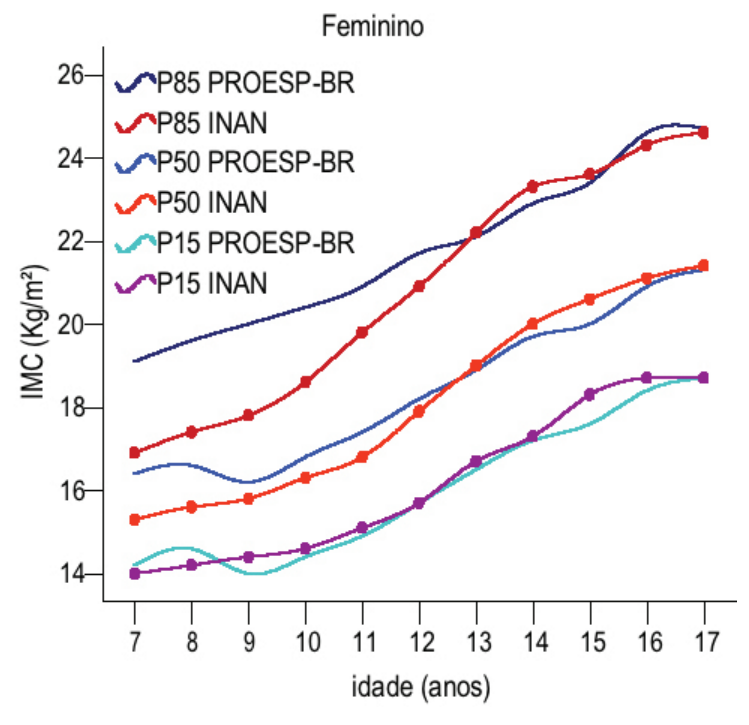

Figura 2. Distribuição dos valores (P15, P50 e P85) de índice de massa corporal $\left(\mathrm{kg} / \mathrm{m}^{2}\right)$ da população brasileira feminina de 7 a 17 anos, em 1989 (INAN) e em 2004/2005 (PROESP-BR). 


\section{DISCUSSÃO}

É conveniente salientar, logo no início das discussões, que os resultados apresentados anteriormente devem ser analisados com cautela. Mesmo sendo uma comparação entre a distribuição do IMC de crianças e adolescentes de dois estudos que contaram com indivíduos de todo o Brasil, a forma com que estas amostras foram constituídas não foi a mesma, gerando certa fragilidade na comparabilidade das distribuições. Essa característica pode ser considerada como uma limitação do estudo. Contudo, considerando que as amostras dos dois estudos contaram com indivíduos de todos estados do país e que o número amostral é significativo, acreditamos ser possível esta comparação se constituir como um indicador de tendência secular do IMC de crianças e adolescentes brasileiros.

Estudos sobre tendência secular em geral e sobre tendência secular do IMC, em especial, são raros no Brasil. Alguns preciosos esforços foram realizados nas regiões Sudeste e Nordeste do país ${ }^{16}$, na cidade de Erechim/RS ${ }^{17}$, em conjunto com a China, Rússia e EUA ${ }^{18}$, e no Nordeste do país ${ }^{19}$. Contudo, no primeiro estudo, foram analisadas crianças de zero a cinco anos de idade; no segundo, foram analisados escolares de 11 a 15 anos de apenas uma escola de ensino público; no terceiro, foram analisadas crianças e adolescentes de levantamentos realizados em 1974 e 1997, apenas das regiões Nordeste e Sudeste; e no último, foram estudados apenas adolescentes do sexo masculino que se apresentaram ao Exército Brasileiro para prestar serviço militar obrigatório nos estados do Nordeste do Brasil. Desta forma, os achados do presente estudo além de apresentar dados mais recentes sobre o IMC, representam as primeiras informações acerca das mudanças na distribuição do IMC da população jovem brasileira de todo o país.

Os resultados indicaram um claro aumento do IMC nos valores de tendência central e do percentil mais alto da distribuição (P85), sendo para as meninas apenas até os 12/13 anos, e nos meninos ao longo de todas as idades estudadas. Estes aumentos foram mais acentuados no P85, indicando que, atualmente, as crianças e adolescentes com valores de IMC mais altos, possuem valores mais altos que as crianças e adolescentes com valores mais altos de IMC do final da década de 80 . Isto sugere que a prevalência de sobrepeso e obesidade aumentou no Brasil. Além disto, como os valores do percentil mais baixo da distribuição (P15) praticamente não apresentaram diferenças entre os levantamentos do INAN e do PROESP-BR, nota-se claramente um distanciamento entre as curvas de distribuição do P50 e do P85, em relação ao P15, no levantamento realizado pelo PROESP-BR.

Este aumento nos valores de IMC da população jovem brasileira parece seguir o mesmo caminho das populações jovens de paises de todo o mundo. Nos EUA, o aumento da gordura corporal vem sendo registrado desde a década de oitenta. Pate et al. ${ }^{20}$ e Ross et al. ${ }^{21}$, comparando os dados NCYFS I e II com os dados do NHES II realizado na década de sessenta, encontraram um claro aumento no somatório de dobras cutâneas de crianças e adolescentes americanos. Em estudo mais recente, Troiano e Flegal ${ }^{12}$, compararam a prevalência e a tendência do sobrepeso de crianças e adolescentes de 7 a 17 anos dos EUA, de 1964 para 1994, encontrando um significativo aumento. Os resultados deste estudo em relação à distribuição do IMC foram bastante similares ao do presente estudo. Em ambos, as maiores mudanças nas distribuições do IMC ocorreram na parte superior das distribuições. Mudanças nos valores de IMC também são reportadas no Canadá ${ }^{22}$. Comparando dados de 1981 com dados de 1996, os autores demonstraram um aumento evidente nos níveis de IMC de meninos e meninas. Semelhante aos resultados do presente estudo, Tremblay e Willms ${ }^{22}$ também reportaram maiores diferenças em idades mais baixas, entre os 6 e 11 anos.

Na Europa, alguns estudos também têm mostrado o aumento nos níveis de IMC da população jovem. Analisando adolescentes de 12 a 18 anos de levantamentos realizados em 1969 e 1996, na Bélgica, Hulens et al. ${ }^{23}$, constataram aumentos na distribuições de valores dos percentis 85 e 90 da amostra de 1996 em relação à de 1969, resultados bastante próximos aos encontrados no presente estudo. Estudando também as diferenças nos valores de IMC de crianças em diferentes períodos, Herpertz et al. ${ }^{24}$, encontraram aumentos nos valores médios de IMC de pré-escolares alemães de 1968 para 1999. Com amostras representativas de adolescentes finlandeses de 1977 e de 1999, Kautiainen et $\mathrm{al} . .^{25}$, observaram aumentos nos valores de IMC nos dois sexos e em todas as idades.

$\mathrm{Na}$ Ásia e na Oceania, também há evidências de aumentos nos valores de IMC de crianças e adolescentes. No Japão, foi evidenciado um marcado aumento dos níveis de IMC de crianças e adolescentes de 6 a 14 anos de 1976 a 200026. Resultados semelhantes foram encontrados na China por Wang et al. ${ }^{19}$, estudando crianças e adolescentes do Longitudinal China Health and Nutrition Surveys conduzi- 
dos em 1991 e 1997. Na Austrália, Olds e Harten²7 sumarizaram estudos sobre os valores de estatura, massa corporal e dobras cutâneas de 41 estudos realizados com crianças e adolescentes de 5 a 17 anos no período de 1899 a 1999. Os autores encontraram aumentos em todas as variáveis ao longo dos anos, mas com especial atenção aos maiores aumentos nos valores de massa corporal nos percentis mais altos, em comparação aos mais baixos, principalmente, a partir da década de 80 , semelhante ao encontrado no presente estudo em relação ao IMC, indicando um possível aumento na prevalência de sobrepeso e obesidade desta população.

Quando os resultados do presente estudo são comparados às pesquisas realizadas no Brasil, notamos certa similaridade nos achados. O estudo desenvolvido por Glaner ${ }^{17}$, apresentou aumento significativo nos níveis médios de IMC de escolares de 11 a 15 anos, de Erechim/RS, com as análises sem a estratificação por idade. Contudo, quando as comparações entre o IMC dos escolares de 1976 são feitas com os escolares de 1996, em cada idade, os aumentos não são tão evidentes. Os resultados de nosso estudo referentes ao P50 também apresentam diferenças marcadas em algumas idades, mas em outras, valores similares entre os anos de 1989 e 2004/2005.

Os resultados do presente estudo, bem como de todos os outros estudos utilizados nesta discussão, demonstram que o número de crianças e adolescentes com sobrepeso e obesidade vem aumentando com o passar dos anos em todo mundo. Considerando que o IMC é uma variável de boa estabilidade e que desta forma crianças obesas possuem maiores chances de se tornarem adultos obesos ${ }^{28,29}$, podemos supor que, possivelmente, nos próximos 10, 15 e 20 anos, o número de adultos obesos aumente ainda mais no mundo inteiro. Esta previsão reforça a já existente preocupação com a obesidade e os problemas a ela associados, e demonstra que as medidas que estão sendo tomadas para controlá-la parecem não estar funcionando, visto que, pelos resultados dos estudos, os níveis de IMC das populações jovens continuam aumentando. Estas informações são preocupantes e é de fundamental importância que novas ações sejam tomadas para que se possa controlar esta epidemia.

\section{CONCLUSÃO}

O presente estudo observou o aumento nos níveis de IMC da população jovem brasileira. Mostrou que a parte superior das distribuições (P85) teve mudanças mais pronunciadas, que as idades mais baixas foram mais afetadas, que os meninos apresentaram aumentos ao longo de todas as idades e as meninas apenas até em torno dos 12/13 anos. Adicionado aos já inúmeros programas e iniciativas contra o aumento da obesidade existentes no Brasil, estes números reforçam a necessidade de políticas públicas para o controle desta epidemia. Este estudo abre caminho para estudos futuros, como a tendência na prevalência do sobrepeso e da obesidade no Brasil, e estudos estratificados por regiões e condições socioeconômicas.

\section{Agradecimentos}

Os autores agradecem ao Ministério do Esporte, ao Conselho Nacional de Desenvolvimento Científico e Tecnológico (CNPQ) e a Fundação de Amparo à Pesquisa do Estado do Rio Grande do Sul (FAPERGS) as inestimáveis contribuições para a execução do Projeto Esporte Brasil (PROESP-BR) em geral e desse artigo em específico.

\section{REFERÊNCIAS BIBLIOGRÁFICAS}

1. Flegal KM, Carroll MD, Kuczmarski RJ, Johnson CL. Overweight and obesity in the United States: prevalence and trends, 1960-94. Int J Obes Met Dis 1998;22(1):39-47.

2. Ogden CL, Troiano RP, Briefel RR, Kuczmarski RJ, Flegal KM, Johnson CL. Prevalence of Overweight Among Preschool Children in the United States, 1971 Through 1994. Pediatrics 1997;99(4):1-7.

3. Troiano RP, Flegal KM, Kuczmarski RJ, Campbell SM, Johnson CL. Overweight prevalence and trends for children and adolescents. The National Health and Nutrition Examination Surveys, 1963 to 1991. Arch Pediatr Adolesc Med 1995;149(10):1085-1091.

4. Cook S, Weitzmen M, Auinger P, Nguyen M, Dietz WH. Prevalence of a metabolic syndrome phenotype in adolescents. Arch Pediatr Adolesc Med 2003;157(8):821-827.

5. Davis CL, Flickinger B, Moore D, Bassali R, Baxter $\mathrm{SD}$, Yin Z. Prevalence of cardiovascular risk factors in schoolchildren in a rural Georgia community. Am J Med Sci 2005;330(2):53-59.

6. Duncan GE, Li SM, Zhou X-H. Prevalence and Trends of a Metabolic Syndrome Phenotype Among U.S. Adolescents, 1999-2000. Diab Care 2004;27(10):2438-2443.

7. Abrantes MM, Lamounier JA, Colosimo EA. Prevalência de Sobrepeso e Obesidade de Crianças e Adolescentes das Regiões Sudeste e Nordeste. J Pediatr 2002;78(4):335-340.

8. Giugliano R, Melo ALP. Diagnóstico de sobrepeso e obesidade em escolares: utilização do índice de massa corporal segundo padrão internacional. J Pediatr 2004;80(2):129-34

9. Bergmann GG, Lorenzi T, Garllip D, Marques A, Araújo MLB, Lemos A, et al. Aptidão Física Relacionada à 
Saúde de Escolares do Estado do Rio Grande do Sul. Perfil 2005;7(7):12-21.

10. Terres NG, Pinheiro RT, Horta BL, Pinheiro KAM, Horta LL. Prevalência e fatores associados ao sobrepeso e à obesidade em adolescentes. Rev Saúde Pública 2006;40(4):2-7.

11. Troiano RP, Flegal KM. Overweight Children and Adolescents: Description, Epidemiology, and Demographics. Pediatrics 1998;101(3):497-504.

12. Wedderkopp N, Froberg K, Hansen HS, Andersen LB. Secular trends in physical fitness and obesity in Danish 9-year-oldgirls and boys: Odense School Child Study and Danish substudy of the European Youth Heart Study. Scand J Med Sci Sports 2004;14(3):150-155.

13. Westerstahl M, Barnekov-Bergkvist M, Hedberg G, Jansson E. Secular Trends in Body Dimensions and Physical Fitness among adolescents in Sweden From 1974 to 1995. Scand J Med Sci Sports 2003;13(2):128-137.

14. INAN - Instituto Nacional de Alimentação e Nutrição. Pesquisa Nacional sobre Saúde e Nutrição: Perfil de crescimento da população brasileira de 0 a 25 anos. Brasília: Ministério da Saúde; 1990.

15. Anjos LA, Veiga GV, Castro IRR. Distribuição dos valores do índice de massa corporal da população brasileira até 25 anos. Rev Panam Salud Publica 1998;3(3):164-173.

16. Monteiro CA, Conde WL. A tendência secular da obesidade segundo estratos sociais: Nordeste e Sudeste do Brasil, 1975-1989-1997. Arq Bras Endocr Met 1999;43(3):186-194.

17. Glaner MF. Tendência secular do crescimento físico e índice de massa corporal em escolares. Rev Min Educ Física 1998;6(2):59-69.

18. Wang Y, Monteiro C, Popkin BM. Trends of obesity and underweight in older children and adolescents in the United States, Brazil, China, and Russia. Am J Clin Nutr. 2002;75(6):971-977.

19. Vasconcelos VL, da Silva GAP. Prevalências de sobrepeso e obesidade em adolescentes masculinos, no Nordeste do Brasil, 1980-2000. Cad Saúde Pública. 2003;19(5):1445-1451.

20. Ross JG, Gilbert GG. The National Children and Youth Fitness Study: A Summary of Findings. JOPERD 1985;56(1):45-50.
21. Ross JG, Pate RR. The National Children and Youth Fitness Study II: A Summary of Findings. JOPERD 1987;58(10):51-56.

22. Tremblay MS, Willms JD. Secular trends in the body mass index of Canadian children. Can Med Assoc J 2000;163(11):1429-1433.

23. Hulens M, Beunen G, Claessens AL, Lefevre J, Thomis M, Philippaerts et al. Trends in BMI among Belgian children, adolescents and adults from 1969 to 1996. Int J Obes 2001;25(3): 395-399.

24. Herpertz-Dahlmann B, Geller F, Böhle C, Khalil C, Trost-Brinkhues G, Ziegler A, et al. Secular trends in body mass index measurements in preschool children from the City of Aachen, Germany. Eur J Pediatr 2003;162(2):104-109.

25. Kautiainen S, Rimpelä A, Vikat A, Virtanen SM. Secular trends in overweight and obesity among Finnish adolescents in 1977-1999. Int J Obes 2002;26(4): 544-552.

26. Matsushita Y, Yoshiike N, Kaneda F, Yoshita K, Kimoto $\mathrm{H}$. Trends in childhood obesity in Japan over the last 25 years from the National Nutrition Survey. Obes Res 2004;12(2):205-214.

27. Olds TS, Harten NR. One Hundred Years of Growth: The Evolution of Height, Mass, and Body Composition in Australian Children, 1899-1999. Hum Biol 2001;73(5):727-738.

28. Guo SS, Chumlea WC. Tracking of body mass index in children in relation to overweight in adulthood. Am J Clin Nutr 1999;70(1):145S-148S.

29. Wang Y, Ge K, Popkin BM. Tracking of body mass index from childhood to adolescence: a 6-y follow-up study in China. Am J Clin Nutr 2000;72(4):1018-1024.
Endereço para correspondência
Gabriel Gustavo Bergmann
Rua das Hortênsias, 465, Igara II.
CEP: 92410580. Canoas, RS. Brazil.
E-mail: gabrielgbergmann@gmail.com 